\title{
The effect of preoperative anemia on postoperative morbidity and mortality in patients undergoing thoracic surgery
}

\author{
Ayten Saracoglu', Didem Güngörr2, Tunç Laçin², Hasan Fevzi Batırel², \\ Mustafa Yüksel' ${ }^{2}$, Zuhal Aykaç' \\ Department of Anesthesiology and Intensive Care', Thoracic Surgery ${ }^{2}$, \\ Marmara University Medical School, Istanbul, Turkey
}

\begin{abstract}
Background and Goal of Study:
Anemia is common in patients undergoing thoracic surgery due to three main risk factors including iron deficiency anemia, chronic disease anemia, iatrogenic anemia ( $I)$, all of which can be prevented and treated.

The incidence of anemia was found as $26 \%$ in a multicenter study involving patients undergoing cardiac surgery, which increased to $47 \%$ in patients having elective vascular surgery (2). However data on the incidence of preoperative anemia for thoracic surgery are limited (3). We hypotized that the incidance of anemia may be higher than expected, which may be associated with both perioperative complications.
\end{abstract}

After obtaining the instutional board approval, this retrospective study enrolled 107 patients older than 18 years who underwent thoracic surgery by a thoracotomy or thoracoscopy between January 2016 and July 2017 . We recorded demographic features, preand postoperative hemograms and other laboratory tests, comorbidities, the transfusions of blood and blood products, complications, mortality, type of surgery, rate of re-exploration, length of ICU and hospital stay. The patients were classified into two groups according to the presence or absence of anemia. The anemia was defined according to the WHO criteria as a hemoglobin level < $12 \mathrm{~g} / \mathrm{dL}$ in woman and $<13,0 \mathrm{~g} / \mathrm{dL}$ in man (4).

\section{Results:}

The incidence of anemia was $\mathbf{4 3 . 9} \%$, being higher in men with $\mathbf{5 7 , 4 \%}$. Compared with patients without anemia, patients with anemia had a significantly increased length of hospital stay, higher rate of ICU admission, higher levels of pre- and postoperative creatinine and lower levels of postoperative $\mathrm{Hb}$ and Htc and lower value of preoperative INR $(p<0.05$, Table I).

The patients who required ICU admission significantly differed from those who did not in low levels of preoperative Hb, pre- and postoperative Htc and increased duration of operation, hospital stay and intraoperative ES use $(p<0.05$, Table 2$)$.

\begin{tabular}{|c|c|c|c|c|c|c|c|c|c|c|c|}
\hline \multicolumn{6}{|c|}{$\begin{array}{l}\text { Table I: Comparison of patient demographics, } \\
\text { type of surgery, the duration of hospital and ICU } \\
\text { stay, parameters for perioperative hematologic } \\
\text { evaluation, the requirement for blood products and } \\
\text { postoperative complications between patients with }\end{array}$} & \multicolumn{6}{|c|}{$\begin{array}{l}\text { Table 2: Comparison of patient outcomes and } \\
\text { postoperative complications between UCU and } \\
\text { ward patients. }\end{array}$} \\
\hline & \multicolumn{2}{|c|}{ Anemia $(-)$} & \multicolumn{2}{|c|}{ Anemia $(+)$} & \multirow[b]{2}{*}{$p$} & & \multirow{2}{*}{\multicolumn{2}{|c|}{ ICU stay (-) }} & \multirow{2}{*}{\multicolumn{2}{|c|}{ ICU stay $(+)$}} & \\
\hline & $\begin{array}{l}\text { meantssd/n}(\%) \\
\end{array}$ & Median & meantsd/n(\%) & Median & & & & & & & \\
\hline Age & $661,37 \pm 12,20$ & 62,50 & $60,89 \pm 13,79$ & 62,00 & $0,967 \mathrm{~m}$ & & meantsdon(\%) & Median & meanisdin $(\%)$ & Median & \\
\hline Female & $27(45,0 \%)$ & & $20(42,6 \%)$ & & & Age & $61,78 \pm 10,61$ & 62,00 & $59,76 \pm 16,99$ & 62,00 & $0,9577^{m}$ \\
\hline Gender Male & $33(55,0 \%)$ & & $27(57,4 \%)$ & & 0,800 & Gender Female & $34(45,9 \%)$ & & $13(39,4 \%$ ) & & \\
\hline BMI & $26,19 \pm 4,11$ & 26,10 & $25,11 \pm 5,03$ & 25,80 & $0,855 \mathrm{~m}$ & Male & $40(54,1 \%)$ & & $20(60,6 \%)$ & & \\
\hline ASA & $2,87 \pm 1,14$ & 3,00 & $2,91 \pm 1,14$ & 3,00 & $0,805 \mathrm{~m}$ & BMI & $26,144,07$ & & $25,265,05$ & & $0,72{ }^{m}$ \\
\hline Decortication & $7(11,7 \%)$ & & $3(6,4 \%)$ & & & ASA & $2,84 \pm \pm 1,18$ & 3,00 & $3,00 \pm 1,03$ & 3,00 & $0,499=$ \\
\hline Type of Lobectomy & $46(76,7 \%)$ & & $36(76,6 \%)$ & & & Decortication & $6(8,1 \%)$ & & $4(12,1 \%)$ & & \\
\hline surgery Pneumonectomy & $3(5,0 \%)$ & & $5(10,6 \%)$ & & $9 !$ & Type of Lobectony & $60(81,1 \%)$ & & $22(66,7 \%)$ & & \\
\hline Sleeve resection & $4(6,7 \%)$ & & $3(6,4 \%)$ & & & surgery Pneumonectomy & $3(4,1 \%)$ & & $5(15,2 \%)$ & & \\
\hline Duration of surgery (b) & $4,14 \pm 1,43$ & 4,00 & $4,07 \pm 1,57$ & 4,00 & $0,663 \approx$ & Sleeve resection & $5(6,8 \%)$ & & $2(6,1 \%)$ & & \\
\hline Hospital stay (days) & $7,08 \pm 2,98$ & 6,00 & $9,96 \pm 5,81$ & 9,00 & $0,011 \mathrm{~m}$ & Duration of surgery (b) & $3,72 \pm 1,26$ & 4,00 & $4,98 \pm 1,60$ & 4,50 & $0,000=$ \\
\hline ICU stay (days) & $12(20,0 \%)$ & & $21(44,7 \%)$ & & $0,006{ }^{x}$ & Hospital stay (days) & $6,88 \pm 3,26$ & 6,00 & $11,644,59$ & 11,00 & $0,000=$ \\
\hline Preoperative Hb (g/dL) & $13,74 \pm 1,07$ & 13,60 & $11,10 \pm 1,36$ & 11,60 & 0,000 m & Preoperative Hb (gddL) & $1,2,89 \pm 1,63$ & 12,90 & $11,89 \pm 1,92$ & 11,90 & $0,008=$ \\
\hline Postoperative Hb (g/dL) & $11,29 \pm 1,54$ & 11,25 & $10,25 \pm 1,66$ & 9,80 & 0,002 m & Postoperative Hb (g/dL) & $10,89 \pm 1,65$ & 11,05 & $10,72 \pm 1,73$ & 10,10 & $0,683=$ \\
\hline Preoperative Htc (\%) & $41,32 \pm 3,26$ & 41,60 & $34,2444,38$ & 35,10 & 0,000 m & Preoperative Htc $(\%)$ & $39,064,75$ & 39,05 & $36,29+5,61$ & 36,50 & $0,009=$ \\
\hline Postoperative Htc (\%) & $34,884,07$ & 34,95 & $30,10 \pm 4,17$ & 29,20 & 0,000 " & Postoperative Htc (\%) & $33,524,4,83$ & 33,80 & $31,3144,29$ & 30,40 & $0,023=$ \\
\hline Preoperative INR & $0,98 \pm 0,22$ & 1,00 & $1,1440,64$ & 1,07 & $0,038=$ & Properative INR & $1,00 \pm 0,18$ & 1,03 & $1,16=0,77$ & 1,06 & $0,236 "$ \\
\hline Postoperative INR & $1,22 \pm 1,30$ & 1,09 & $1,16 \pm 0,18$ & 1,16 & 0,017 m & Postoperative INR & $1,221 \pm, 18$ & 1,11 & $1,13 \pm 0,16$ & $1,1,4$ & $0,369=$ \\
\hline Preoperative Creatinine & $0,82 \pm 0,18$ & 0,82 & $1,36 \pm 4,42$ & 0,69 & $0,006=$ & Preoperative Creatinine & $0,77 \pm 0,20$ & 0,74 & $1,71 \pm 5,26$ & 0,77 & $0,410=$ \\
\hline Postoperative Creatinine & $0,83 \pm 0,29$ & 0,80 & $0,70 \pm 0,26$ & 0,67 & 0,003 " & Postoperative Creatinine & $0,66 \pm 0,25$ & 0,74 & $0,8110,35$ & 0,75 & $0,597=$ \\
\hline Preoperative & $279,0 \pm 101,7$ & 252,5 & $279,9 \pm 114,3$ & 260,0 & $0,770 \mathrm{~m}$ & Preopera & $282,5 \pm 106,8$ & 260,0 & $272,5 \pm 108,4$ & 249,0 & 0,761 \\
\hline $\begin{array}{l}\text { Perioperative bleeding } \\
\text { (mL) }\end{array}$ & $495 \pm 378$ & 400 & $383 \pm 262$ & 360 & 0,155 m & $\begin{array}{l}\text { Perioperative bleding } \\
\text { volume (cc) }\end{array}$ & $421,8 \pm 265,5$ & 395,0 & $499,74455,3$ & 400,0 & \\
\hline Perioperative fluid (mL) & $1385 \pm 6.14$ & 1200 & 13064413 & 1200 & 0,957 in & Perioperative fluid (mL) & $1352 \pm 572$ & 1200 & $1347 \pm 445$ & 1200 & $0,712 "$ \\
\hline $\begin{array}{l}\text { Perioperative urine } \\
\text { output (mL) }\end{array}$ & $417,0 \pm 231,4$ & 400,0 & $405,3 \pm 243,1$ & 375,0 & $0,788 \mathrm{~m}$ & $\begin{array}{l}\text { Perioperafive urine } \\
\text { output(mL) }\end{array}$ & $382,9 \pm 187,8$ & 400,0 & $476,2 \pm 310,5$ & 400,0 & 0,290 \\
\hline Intraoperative RBC (D) & $4(6,7 \%)$ & & $7(14,9 \%)$ & & $0,164 x^{8}$ & Intraoperative RBC & $2(2,7 \%)$ & & $9(27,3 \%)$ & & $0,000{ }^{x}$ \\
\hline & & & & & $0,318 x^{x}$ & Intraoperative FFP & & & & & $0,0866^{x}$ \\
\hline Intraoperative platelet (U) & $0(0,0 \%)$ & & $0(0,0 \%)$ & & & Intraoperative platelet & $0(0,0 \%)$ & & $0(0, C$ & & \\
\hline Postoperative RBC (U) & $7(11,7 \%)$ & & $10(21,3 \%)$ & & $0,177 x^{x}$ & Postoperative RBC & $10(13,5 \%)$ & & $7(21,2,2)$ & & $0,314^{x}$ \\
\hline Postoperative FFP (U) & $0(0,0 \%)$ & & $0(0$, & & . & Postoperative FFP & & & & & . \\
\hline Postoperative Platelet (U) & $0(0,0 \%)$ & & $0(0,0 \%)$ & & . & Postoperative Platelet & $0(0,0 \%)$ & & $0(0,0 \%)$ & & . \\
\hline & $1(1,7 \%)$ & & & & $1,000 \times$ & Postoperative MII & & & & & $0,318^{x}$ \\
\hline Postoperative Arrythmia & $0(0,0 \%)$ & & $0(0,0 \%)$ & & & Postoperative Arrythmin & $0(0,0 \%)$ & & $O(0,0 \%)$ & & . \\
\hline Exitus & $0(0,0 \%)$ & & $0(0,0 \%)$ & & . & & $0(0,0 \%)$ & & $O(0,0 \%)$ & & \\
\hline Re-exploration & $0(0,0 \%)$ & & $2(4,3 \%)$ & & $0,191 x^{x}$ & Reerploration & $1(1,4 \%)$ & & $1(3,0 \%)$ & & $0,524^{x}$ \\
\hline
\end{tabular}

Our findings suggest that the incidence of anemia is high in patients undergoing thoracic surgery. Eventhough the rate of mortality is not affected, anemia is associated with a higher rate of ICU admission and longer hospital stay. Additionally an increased intraoperative use of ES is likely to contribute to morbidity by exposing patients to transfusion-associated complications.

In order to reduce anemia-induced perioperative complications the treatment of anemia during preoperative period should be considered who are candidate for thoracic surgery. 